\title{
The Sphincter Mechanism of the Urinary Bladder and the Urethro
}

\author{
RUSSELL T. WOODBURNE \\ Department of Anatomy, The University of Michigan Medical School, \\ Ann Arbor, Michigan
}

Clinical studies of continence in the female and the determination of means to correct incontinence have raised serious questions concerning existing concepts of muscular arrangements in the bladder and the urethra and the presence and action of a sphincter at the vesical neck. Previously there has been a general assumption that the urinary bladder is structured like the gastrointestinal tract with a two layer musculature having circular and longitudinal orientation. The two-part autonomic nervous system has been related to the bladder in terms of activation and inhibition, a common statement being that the parasympathetic fibers are motor to the detrusor and inhibitory to the internal sphincter and the sympathetic innervation motor to the internal sphincter and inhibitory to the detrusor. Evidence for these statements has never been good and recently the results of many diverse studies are leading to an entirely contrary concept of structure and function in these organs.

Physiological evidence has not supported the assumptions noted above. In 1933, Denny-Brown and Robertson demonstrated that urination is a reflex act mediated through the centers in the sacral cord and in 1936, evidence adduced by Evans denied any part in vesical function by the sympathetic nerves. Langworthy, Kolb and Lewis ('40) also advanced the opinion that the detrusor muscle is devoid of sympathetic innervation. There is documentation for these statements in other studies and it is now certain that the emptying reflex of the urinary bladder is, like the rectum, completely under the control of the parasympathetic nerves which reach it, the parasympathetic reflex arc being completed by afferent neurons which respond to stretch receptors within the mucosa and submucosa (Langworthy and Murphy, '39).
It is now generally recognized that the sympathetic innervation reaches only the representation of ureteral musculature in the trigone of the bladder. It also conducts most of the pain afferents from the bladder.

It will be obvious that current concepts of structure in the urinary bladder and urethra do not harmonize with the physiological evidence and there is clearly a need for a re-examination of this area. Is the bladder composed of a nicely layered musculature? Is it equipped with a discrete and separate vesical or internal urethral sphincter and if such a sphincter exists is it antagonistic in action to the detrusor muscle? If one denies a sphincter at the vesical neck, what mechanism is available to keep the orifice closed as urine accumulates and what mechanism holds the orifice open as vesical pressure drops during micturition? To answer these and other questions a study has been conducted on the structure of the bladder and the urethra in both male and female sexes by a combination of gross and microscopic techniques using both canine and human bladders. Stimulation experiments were also conducted on the dog and embryonic specimens were studied.

It is traditionally stated that the muscular coats of the bladder are arranged in layers, and inner longitudinal, middle circular, and outer longitudinal layers are frequently recognized. Observations in both dog and man on bladders from which the adventitia was stripped away and which were macerated to reduce the binding power of the connective tissue show that these three traditional layers are far from being complete nor have they been accurately described. General views of the exterior of the bladder (figs. 1,2) show a number of longitudinal fascicles but 
through these fascicles appear transverse bundles or bundles in oblique orientation to the surface. Observations made from within the bladder (fig. 1) also show longitudinally running bundles converging on the vesical neck. However, it is clear that the longitudinal bundles seen on both surfaces are in complete continuity with the oblique and transverse fascicles existing throughout the substance of the bladder wall. As early as 1891, Griffiths denied the separate strata in the urinary bladder observing that muscular bundles run from plane to plane and become circular and oblique in the region of the neck of the bladder. More recently, Hunter ('54) analyzed the musculature of the bladder and found that the apparently longitudinal sur-

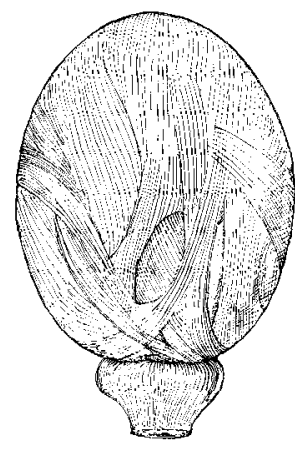

Anterior

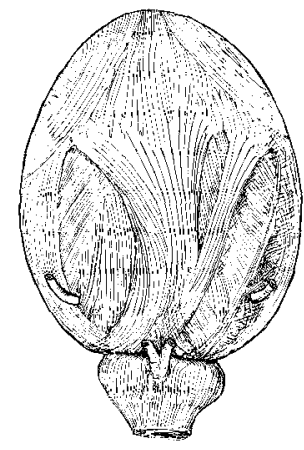

Posterior

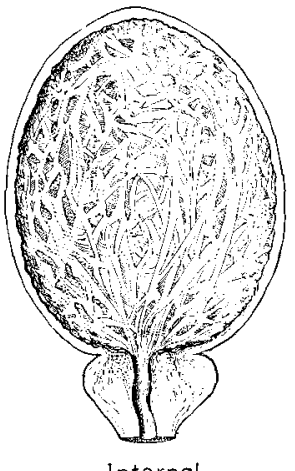

Internal

Fig. 1 The musculature of the human bladder. Note descending longitudinal bundles continuing into prostatic urethra.
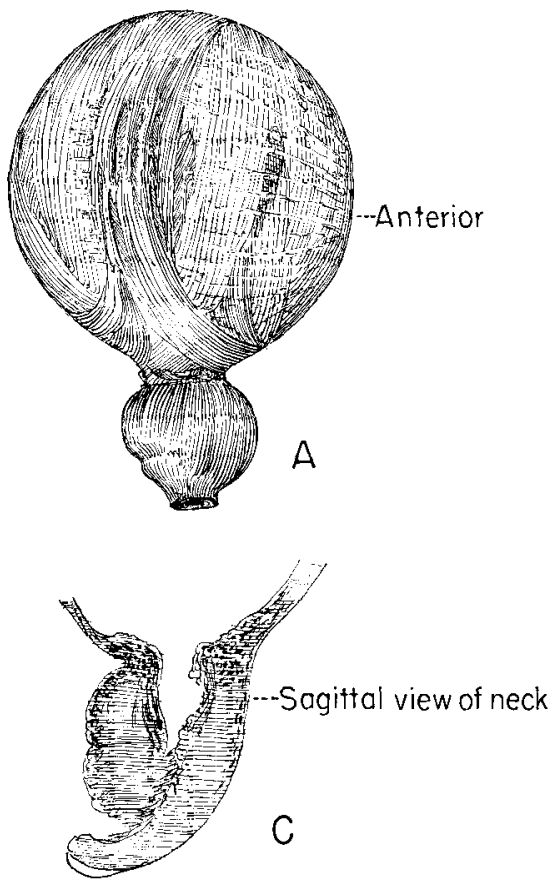
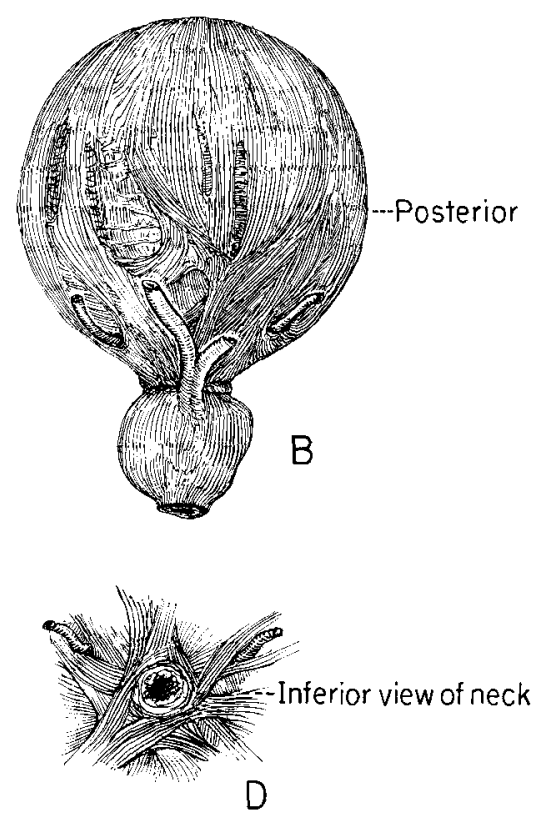

Fig. 2 Views of the musculature of the bladder of the dog: A, anterior aspect; $B$, posterior aspect; $C$, a vertical section of the vesical neck and the prostate gland; $D$, a dissection of the external musculature at the plane of the vesical neck. 
face bundles separated from one another to run deeper into the wall and decussate. He described a maximum of 5 decussations for some fascicles. It is obvious therefore that the muscular coat of the bladder is a meshwork. It consists of fascicles, some broad, some narrow, running in many directions, changing planes and orientation, crisscrossing and decussating through the wall. Generally speaking the vertex is an area in which many of the surface bundles turn inward, but this is not the only point of such penetration.

A region of particular interest in the musculature of the bladder is that of the vesical neck where it is traditionally stated that an accumulation of circularly disposed bundles constitutes an internal urethral sphincter. A true sphincter here has never been established. Wesson, in 1920, felt that he could follow anterior fascicles from the bladder wall which circled behind the urethral orifice and fascicles from the posterior wall of the bladder which circled in front of it. The opposing traction of these muscle bundles was advanced as constituting an internal sphincter. There have been other attempts more recently by Langreder ('56) and Vernet ('60) to follow even more minutely the course of muscle bundles at the vesical neck and some of these efforts have yielded most detailed accounts of spiraling arrangements and alternate longitudinal and circular bundles which are very hard for other investigators to confirm. Denial of an internal urethral sphincter was made as early as 1883, when LeGros Clark stated that he was unable to find any sphincter of annular fibers at the neck of the bladder. Griffiths, in 1891, denied a vesical sphincter, finding no accumulation of circular fibers or any formation that would act as a sphincter at the neck of the bladder. There can be no denial that a minor accumulation of muscle bundles exists about the vesical orifice.

A special study of this region was made by cutting away the urethra from a canine bladder and dissecting under microscopic control the fascicles of muscle which appear to condense around the neck of the bladder. It was instructive to find that the accumulation of muscle in this region does not constitute a sphincter of annular fibers.
Fascicles could be traced into the region of the proximal urethra and certain of these fascicles were seen to swing part way around the opening (fig. 2D). However, it was characteristically noted that these bundles were composed of arching muscle fascicles, fascicles which arched toward and then away from the urethral opening and did not encircle it. Analysis of the traction which would be exerted by such arching fibers convinces one that they would pull away from the center of the opening rather than constrict around it, and therefore should be considered as part of an opening mechanism rather than as a sphincter. These observations were more clearly made in the canine bladder than in the human. They strongly suggest, however, that we do not deal in the vesical neck with a sphincter mechanism at all, but rather that the musculature here is continuous with the detrusor and acts with it and not against it in opening the urethral aperture.

Observations of the interior of the bladder in both canine and human specimens (fig. 1) show that the internal longitudinal fascicles are traceable directly downward into the submucosal muscular layer of the urethra. This is true in both male and female specimens. Clegg ('59) stated that the musculature of the proximal urethra is partly derived from and continuous with the anterior longitudinal muscle of the bladder. He also concluded that there is no anatomical sphincter at the bladder neck. The continuous and identical character of these urethral muscle bundles makes it impossible to regard them as the antagonists of the same bundles in the bladder wall. It is, I think, not well appreciated how thick is the longitudinal muscle of the urethra and how completely it constitutes the innermost and major portion of the muscular wall in both male and female. Representative sections of the male and female urethra are illustrated in figures $3 \mathrm{~A}$ and $\mathrm{B}$. These are in the form of tissue maps, not detailed microscopic drawings, but they make clear that a very large longitudinal segment of musculature lies directly under the submucosa in both male and female specimens and runs throughout the prostatic and membranous urethra in the male and the entire urethra 

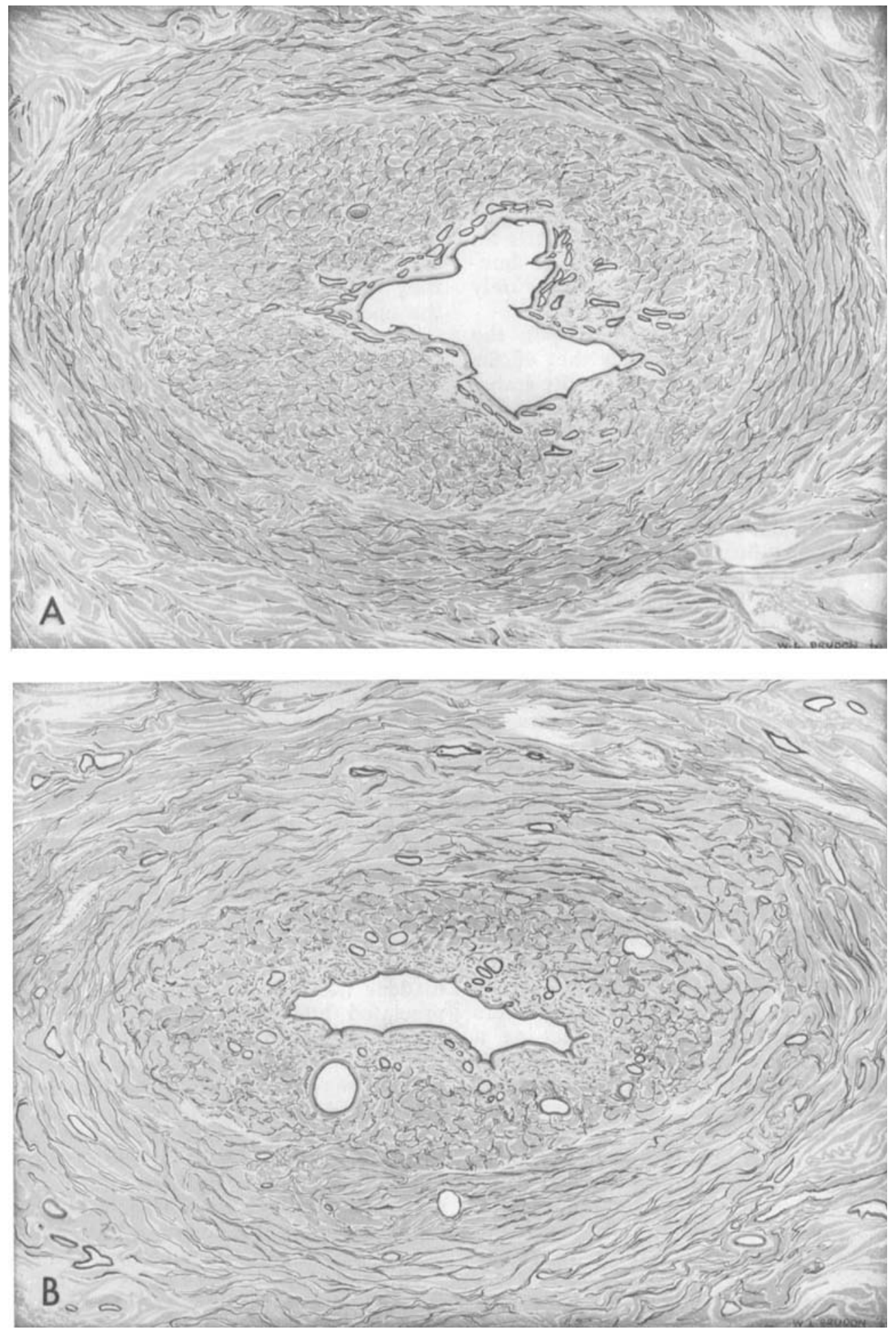

Figure 3 
in the female. This is not the small longitudinal contribution of the ureteral muscle known as Bell's muscle. Bell's muscle is presumably lost in the larger posterior mass of urethral muscle.

If one is to deny the presence of a sphincter at the neck of the bladder, it becomes necessary to consider how closure of the bladder neck is actually effected. It seemed that it might be profitable to look into the existence of the elastic tissue in this area. Accordingly material was cut and sections were stained with combined elastic tissue and muscle stains and the amount of elastic tissue determined. In the general bladder wall elastic tissue was found in small amounts beneath the epithelium and also interspersed among the muscle layers of the wall. Comparison of the situation in the bladder was made with the representation of elastic tissue in sigmoid colon and rectum, and it was found that elastic tissue is somewhat more richly represented in the urinary bladder but not strikingly so.

However, examination of the bladder neck displayed an exceedingly rich collection of elastic tissue. This is shown in a cross section of canine bladder neck in figure 4A. It is apparent here that there is a great concentration of black elastic fibers in the submucosal region and also interspersed among the muscle bundles. Elastic tissue is particularly concentrated among the more centrally lying longitudinally oriented muscle fascicles. The same appearance is seen in figure $4 \mathrm{~B}$ which portrays the comparable region in the human vesical neck. Elastic tissue thus intimately related to muscle fascicles constitutes an intermixture such as warrants the name myoelastic tissue. Examining figures $3 \mathrm{~A}$ and $\mathrm{B}$ of the tissue maps of the urethra, it is apparent that elastic tissue retains the same close relationship to muscle throughout the prostatic and membranous urethra in the male and for the entire length of the urethra in the female. Among the central longitudinally oriented muscle, the elastic tissue is broken up into small segments of generally circularly oriented elas-

Fig. 3 A, A tissue map of a cross-section of the male prostatic urethra. Weigert stain. B, A tissue map of a cross-section of the female prostatic urethra. Weigert stain. tic fibers; among the outer circular muscle, elastic fibers clearly parallel the fascicles of muscle. My evidence indicates that elastic tissue is exceedingly prominent in the neck of the bladder and is represented throughout the urethra in a much higher proportion than is usual in other submucosal and muscular areas.

Certain functional considerations should be adduced from this description of the arrangement of muscular tissue and elastic tissue in the bladder neck and urethra. There is complete continuity of muscle through the bladder and urethra, and there is no break in the bladder neck produced by an annular sphincter which would operate in opposition to the detrusor muscle of the bladder. Embryological considerations give no support for a separate developmental sequence being involved in the origin of the urinary bladder and urethra. Both bladder and urethra arise from the urogenital portion of the cloaca (Arey, '34; Patten, '46), their distinction being based only on the spheroidal enlargement of the proximal part of the allantois which results in the urinary bladder. The constituents of their walls are the same, and the musculature of the bladder extends without interruption as the muscular wall of the urethra (fig. 5). Nor do the studies of nervous innervation of the urinary bladder and urethra cited above lend any support to a separation in structure or function of these parts. There is now general agreement on the role of the nerves of the urinary bladder and understanding of the effects of nerve injury and spinal cord lesions in disturbances of micturition. The old concept that the detrusor is motivated by the parasympathetic nerves and that these nerves are at the same time inhibitory to the internal sphincter and, conversely, that the sympathetic system operates in the opposite way must be discarded.

In order to study the response of the urethra to bladder stimulation, the urethra of a dog was placed under direct observation. We stimulated the bladder muscle above the vesical neck and observed strong contraction of the longitudinal muscle of the urethra which coincided with that of the detrusor muscle of the bladder. The longitudinal muscle of the bladder neck and of the urethra constitutes an opening 

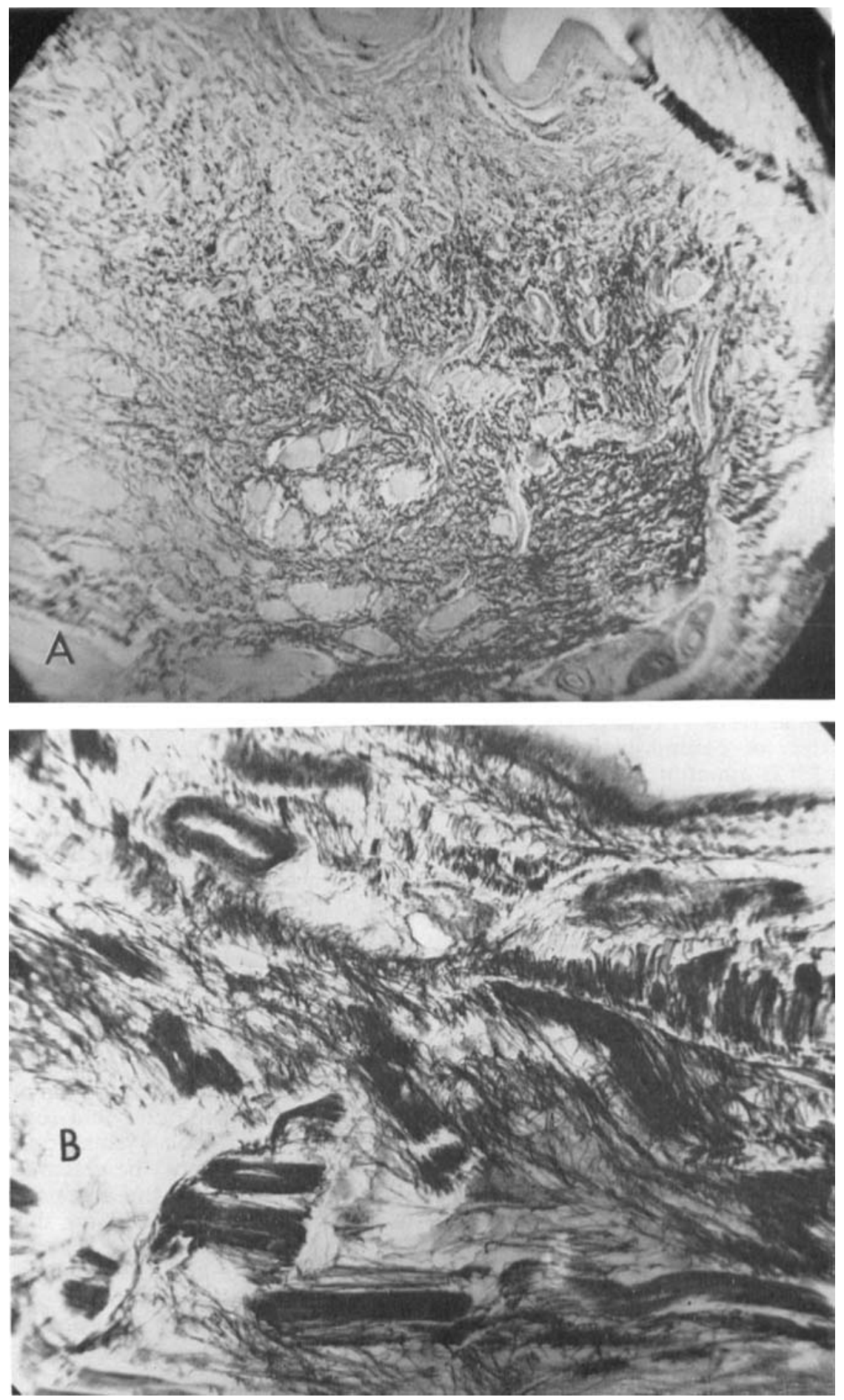

Fig. 4 A, Photomicrograph of a segment of a cross-section of the proximal part of the urethra in the dog. Weigert stain, $\times 40$. B, Photomicrograph of a segment of a cross-section of the submucosal area of the proximal urethra in man. Weigert stain, $\times 50$. 


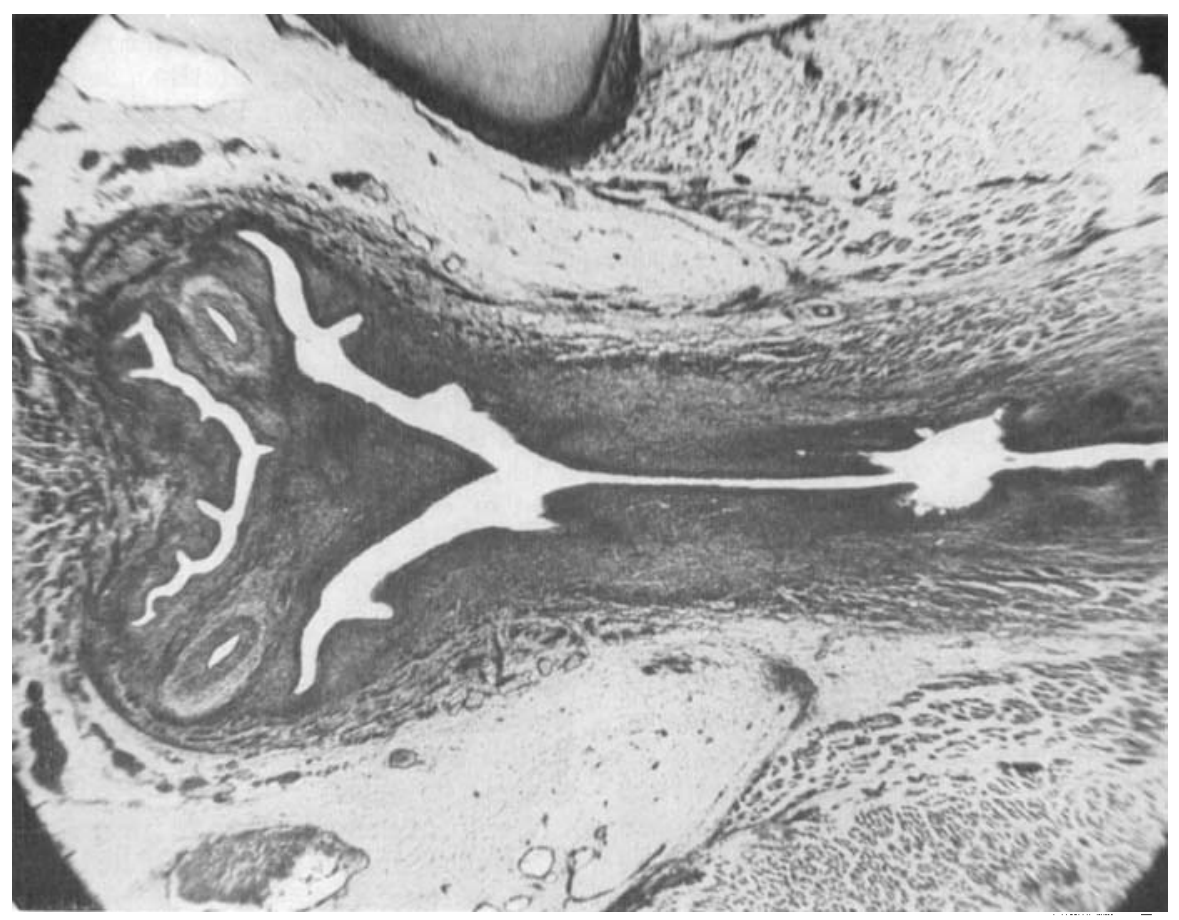

Fig. 5 Photomicrograph of a longitudinal section of bladder and urethra of $86 \mathrm{~mm}$ embryo. Masson stain, $\times 25$.

mechanism for the neck of the bladder. In this action there is a distinct shortening of the urethra coupled with a funneling of the vesical neck and opening of the aperture of the urethra. With the bladder globular from distention, its longitudinal bundles pull radially and open the vesical orifices by direct outward action. Several observers, Scher ('50) and Lapides ('58), have noted cystoscopically that the normal bladder opens by widening of the vesical neck and shortening of the urethra. It should be clear then that the accumulation of muscle at the bladder neck, which has been regarded as a vesical or internal urethral sphincter, does not actually constitute a sphincter at all. The musculature here is not separate from the rest of the bladder musculature, and it contracts with and not against the detrusor. It should be considered as serving in the emptying mechanism as a special device to shorten the urethra and to aid in opening the vesical orifice. This action, coupled with the hydrostatic pressure being imposed by the contraction of the detrusor complex, pro- vides for the initiation of micturition. Such a mechanism obviously does not require an inhibiting sympathetic innervation in the region of the neck of the bladder.

The solution of the role of the musculature of the vesical neck and the urethra and its correlation with the detrusor muscle under the control of one innervation, the parasympathetic, explains the active phase of micturition, but leaves obscure the means whereby the bladder retains a considerable volume of fluid without leakage during its inactive period. It is apparent that there must be some mechanism for holding the orifice of the vesical neck closed as the bladder expands and its muscular wall is stretched. Our investigations of elastic tissue at the vesical neck and in the urethra give the clue to this function. The elastic tissue has been seen to be generally circular in orientation; it is especially prominent at the vesical neck, and it continues in a relatively high concentration throughout the female urethra and the posterior urethra of the male. 
Bodian ('57), also stained sections of blocks of prostatic urethra and noted a preponderance of elastic tissue in this portion of the urethra.

In the control of the hollow tubes of the body, one must be aware of the pertinence of Laplace's law. This law states that, when in equilibrium, pressure exerted on the wall of a tube by its fluid contents is directly proportional to the tension in the wall in relation to its length and inversely proportional to the radius of the tube. The relationship is expressed in the formula $\mathrm{P}=\mathrm{T} / \mathrm{r}$. An analysis of Laplace's law as applied to blood vessels has been made by Burton ('51). He noted that for such tubular structures, tension in the wall in dynes per centimeter of length consists of elastic tension due to elastic tissue and the elasticity of the other components of the wall, active tension due to the contraction of the smooth muscle of the wall, and interfacial tension represented by surface tension of the contained fluid. He noted that maintenance tension in blood vessels is well correlated with the presence and amount of elastic tissue in their walls and that large and medium sized vessels require good representation of this material. It is clear that, like the larger blood vessels, the urethra and the bladder neck utilize elastic tissue to maintain a high $T$ value. Contributing to the total $\mathrm{T}$ here will be the tension exerted by the smooth muscle of the wall and its other constituents. Observation of the formula also shows that with a very small radius of lumen, as would be true in the collapsed urethra, the $\mathrm{T}$ over $\mathrm{r}$ quotient is high, and very large pressures would be resisted. Possible contributors to a perfectly closed lumen are the venous channels which lie directly under the epithelium. These channels, filled with blood, provide a soft packing material which could be important in more intimate approximation of the walls and thus in complete and final closure of the lumen. As the lumen is opened, the radius of the lumen rises. The $\mathrm{T} / \mathrm{r}$ quotient is then much lower and the urethra will remain open under conditions in which pressure in the bladder is dropping.

Of much more importance is the length of the urethra. This is predicted in the formula where the $T$ value is represented as in dynes per centimeter of length. The greater the length the greater is the total $T$. By serial sectioning of the urethra in the dog, Lapides ('58), has strikingly shown that this tube, completely competent in the beginning, can be made progressively incompetent by removing it in parts. He also demonstrated that, by placing gentle traction on the urethra and elongating it, the tube is more continent than before. He reports that a $3-\mathrm{cm}$ length of urethra is a critical requirement for continence in the human female. This emphasizes the statements which have been made above to the effect that micturition follows a shortening and funneling of the urethra. This funneling by direct muscle action obviously enlarges the lumen at the upper end and the shortening has a direct effect in reducing tension due to length. Thus the normal act of micturition is one in which both tension is reduced and the lumen is enlarged.

Length in the urethra is increased by the normal activities of the pelvic diaphragm and of the urogenital diaphragm. The pelvic diaphragm (fig. 6), exerts an upward traction on the pelvic viscera and comes into tonic contracture in the erect posture. Its musculature can, of course, also be contracted on a voluntary basis in any position. The effect of the action of the pelvic diaphragm is to elevate the urinary bladder and to stretch the urethra in either sex. A lengthening effect by downward traction is produced by the musculature of the urogenital diaphragm (fig. 6). This diaphragm, represented particularly as the external sphincter muscle of the urethra, is the region of greatest resistance in the entire female urethra and in the posterior urethra in the male. At this point the urethra is narrowest and is kept firmly collapsed, except during micturition, by the enclosing strands of the external sphincter muscle. These fascicles arch from side to side and, between them, produce an anteroposterior collapse of the urethra. In the external sphincter there is also a group of fibers which arches in front of the urethra and then extends fairly far back on the ischial ramus to attach to bone (Oelrich, unpublished). Since the urogenital diaphragm slopes downward and backward in the 


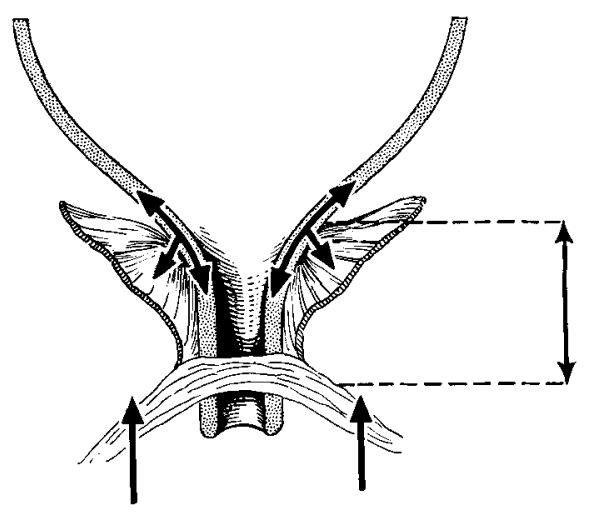

VOIDING:

Perineal and pelvic muscles relaxed

Detrusor muscle active

Elastic tissue stretched

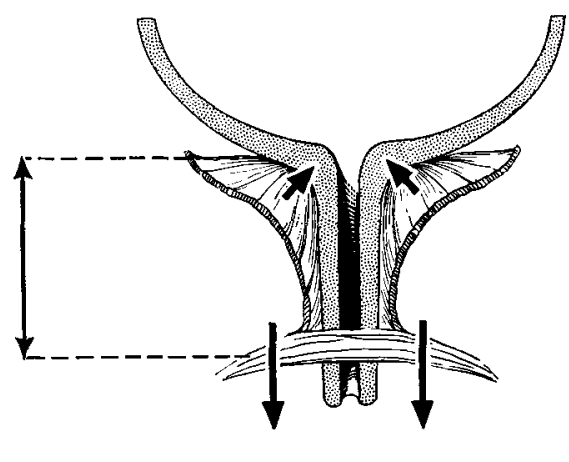

FILLING:

Detrusor muscle inactive

Elastic tissue effective

Perineal and pelvic muscle in tone

Fig. 6 Schema of relations in voiding and filling.

erect posture, this muscle not only compresses the urethra but also elongates it. Thus, between the upward pull of the pelvic diaphragm and the downward pull of the perineal muscles, the urethra is increased in length in the rest position (fig. $6)$.

It is clear that we must abandon the notion that there is a sphincter at the vesical neck. Indeed, we must adopt the contrary view that opening of the urethra is initiated at the vesical neck by the concurrent contraction of the detrusor muscle of the urinary bladder and of the longitudinal fascicles of smooth muscle which descend into and make up a large part of the muscular wall of the urethra. The contraction of this musculature under the same innervation and at the same time as the detrusor muscle of the bladder results in a shortening and funneling of the urethra, so that the lumen of the urethra is opened proximally and the tube as a whole is materially shortened. Greater recognition is warranted for the large amount of elastic tissue which appears in the region of the vesical neck and throughout the urethra and which doubtless exerts its influence in constricting the lumen of the tube and maintaining closure. Consideration of both the anatomy and the law governing tubular structures of the body shows that the entire urethra in the female and the prostatic and membranous parts of the urethra in the male constitute the sphincter of the bladder in each sex. The external sphincter of the urethra plays a role in maintaining the greatest narrowing of the tract in both sexes and also participates in the lengthening of the tube in conjunction with the musculature of the pelvic diaphragm. Maintenance of closure of the urethra, and thus continence, are contributed to by factors which reduce the lumen of the tube and increase its length (fig. 6).

\section{SUMMARY}

A number of revisions are required in the concepts of anatomists and clinicians with respect to the structure and function of the urinary bladder and the urethra. There is evidence that no actual vesical or internal urethral sphincter exists and that no sphincteric function is served in the region of the neck of the bladder. The entire urethra in the female and the prostatic and membranous urethra in the male is the sphincter of the bladder. Closure of the urethra is greatly assisted by the exceptional development of elastic tissue at the bladder neck and along the 
length of the urethra, such elastic tissue being most prominent submucosally and intermingled with the innermost muscle bundles. Micturition is accomplished by the pressure exerted by the detrusor muscle (the entire muscular complex of the bladder) assisted by its continuous urethral musculature. The special contribution of the muscle of the bladder neck and urethra to the opening mechanism is in shortening and widening the urethra and in opening the aperture of the vesical neck in a funnel-like widening. The control of micturition according to the concepts of structure outlined above can be achieved by a simple reflex and by the single (parasympathetic) innervation as has been established in the neurophysiology of the organ.

\section{LITERATURE CITED}

Arey, L. B. 1934 Developmental Anatomy. W. B. Saunders Co., Philadelphia.

Bodian, M. 1957 Some observations on the pathology of "congenital idiopathic bladderneck obstruction" (Marion's disease). Brit. J. Urol., 29: 393.

Burton, A. C. 1951 On the physical equilibrium of small blood vessels. Am. J. Physiol., 164: 319.

Clegg, E. J. 1959 The musculature of the human prostatic urethra. J. Anat., 91: 345.
Denny-Brown, D., and E. G. Robertson 1933 On the physiology of micturition. Brain, 56: 149.

Evans, J. P. 1936 Observations on the nerves of supply to the bladder and urethra of the cat with a study of their action potentials. J. Physiol., 86: 396.

Griffiths, J. 1891 Observations on the urinary bladder and urethra. J. Anat. Physiol., 25: 549.

Hunter, DeW. T., Jr. 1954 A new concept of urinary bladder musculature. J. Urol., 71; 695 .

Langreder, W. 1956 Die weibliche Urethra, Zbl. Gynäk., 78: 561 .

Langworthy, O. R., L. C. Kolb and L. G. Lewis 1940 Physiology of Micturition. The Williams and Wilkins Co., Baltimore,

Langworthy, O. R., and E. L. Murphy 1939 Nerve endings in the urinary bladder. J. Comp. Neur., 71: 487.

Lapides, J. 1958 Structure and function of the internal vesical sphincter. J. Urol., 80: 341.

LeGros Clark, E. 1883 Some remarks on the ' anatomy and physiology of the urinary bladder and the sphincter of the rectum. J. Anat. Physiol., 17: 442 .

Oelrich, T. M. Unpublished observations.

Patten, B. M. 1946 Human Embryology. The Blakiston Co., Philadelphia.

Scher, S. 1950 Some observations on the anatomy of the bladder neck and posterior urethra with reference to prostatic obstruction. Brit. J. Urol., 22: 116.

Vernet, G. 1960 Physiologie der Miktion, Z. Urol., 53: 181.

Wesson, M. B. 1920 Anatomical, embryological and physiological studies of the trigone and neck of the bladder. J. Urol., 14: 278. 